\title{
Effect of adipose-derived stem cell-conditioned medium on the proliferation and migration of B16 melanoma cells
}

\author{
JU-HEE LEE $^{1 *}$, CHUL HONG PARK ${ }^{1 *}$, KWANG-HOON CHUN $^{2}$ and SOON-SUN HONG ${ }^{1}$ \\ ${ }^{1}$ Department of Biomedical Sciences, College of Medicine, Inha University, Incheon 400-712; \\ ${ }^{2}$ Gachon Institute of Pharmaceutical Sciences, College of Pharmacy, Gachon University, \\ Incheon 406-799, Republic of Korea
}

Received July 22, 2014; Accepted April 8, 2015

DOI: $10.3892 / 01.2015 .3360$

\begin{abstract}
Adipose-derived stem cells (ASCs) are a population of cells derived from adipose tissue. ASCs exhibit multilineage development potential and are able to secrete various factors, which influence adjacent cells. Previous studies have reported the effectiveness of ASC-conditioned medium (ASC-CM) in wound healing, anti-melanogenesis, wrinkle improvement and hair growth. In the present study, the anticancer function of ASC-CM was investigated in vitro and in vivo. An MTT assay revealed that ASC-CM significantly decreased the proliferation of B16 melanoma cells in a time- and dose-dependent manner $(\mathrm{P}<0.01)$. Cell cycle analysis indicated that ASC-CM significantly increased the number of cells in G1 phase while reducing the number of cells in the $S$ and $\mathrm{G} 2 / \mathrm{M}$ phases $(\mathrm{P}<0.01)$. Furthermore, a wound migration model demonstrated that ASC-CM treatment significantly decreased the migration ability of $\mathrm{B} 16$ melanoma cells $(\mathrm{P}<0.01)$. In addition, C57BL/6 mice were administered with a single intratumoral injection of ASC-CM, daily or every other day, and a significant reduction in the volume of the tumor mass was observed compared with that of the control group $(\mathrm{P}<0.01)$. Thus, the findings of the present study indicated that ASC-CM has an anti-tumorigenic effect on B16 melanoma cells in vitro and in vivo, and may potentially be used to support the treatment of melanoma in the future.
\end{abstract}

Correspondence to: Professor Soon-Sun Hong, Department of Biomedical Sciences, College of Medicine, Inha University, 7-241, 3-ga, Shinheung-dong, Jung-gu, Incheon 400-712, Republic of Korea E-mail: hongs@inha.ac.kr

Professor Kwang-Hoon Chun, Gachon Institute of Pharmaceutical Sciences, College of Pharmacy, Gachon University, 191 Hambakmoero, Yeonsu-gu, Incheon 406-799, Republic of Korea

E-mail:khchun@gachon.ac.kr

*Contributed equally

Key words: melanoma, adipose-derived stem cells, adipose-derived stem cell-conditioned media, B16 melanoma cells

\section{Introduction}

Adipose-derived stem cells (ASCs) comprise a plastic-adherent, proliferative, multipotent cell population isolated from the stromal vascular fraction (SVF) of adipose tissue (1). ASCs are capable of self-renewal and are able to differentiate into various types of cell populations, including adipocytes, osteoblasts, chondrocytes, myoctyes and neurons. Thus, ASCs are valuable sources of stem cells for use in regenerative medicine and cosmetic applications (2). As with embryonic stem cells (ESCs) and induced pluripotent stem cells (iPSCs), ASCs contribute to tissue repair and skin rejuvenation, as well as bone and cartilage formation. These therapeutic effects of ASCs are considered to result from the differentiation of ASCs into multiple cell types, as well as the paracrine action of the diverse cytokines and growth factors secreted by ASCs onto adjacent tissues $(3,4)$.

Although ASCs are considered to be a promising therapeutic tool with various potential clinical applications, the effect of ASCs on tumor growth, metastasis and recurrence are controversial. A large number of studies have indicated that ASCs promote the proliferation of cancer cells. For example, when ASCs were co-injected with tumor cells, they promoted the tumorigenic growth of $\mathrm{H} 460$ human non-small cell lung cancer cells and U87MG malignant glioma cells in nude mice (5). A similar tumorigenic effect was observed in human prostate (6) and breast (7) cancer cells in mice. Furthermore, co-culture of ASCs enhanced the proliferation of human squamous cell carcinoma cells (8) and the migration of breast cancer cells in a xenograft model (9). However, conversely, numerous studies have reported suppressive effects of ASCs on tumorigenesis. For example, human mesenchymal stromal cells were demonstrated to reduce lung metastasis, the growth of breast cancer cells (10), the proliferation of pancreatic cancer cells (11), and LNCaP or PC3 prostate cancer cell-derived tumor growth in mice (12) by inducing cell death.

Melanoma is a malignant tumor of melanocytes. The occurrence or melanoma has increased steadily between 2002 and 2011, with an average incidence increase of $1.4 \%$ per year in the USA (13). Melanoma is a dangerous disease entity if not detected in the early stages, as recurrence is common (14). Linkage analysis has revealed that families with melanoma possess mutations in the $\mathrm{p} 14^{\mathrm{ARF}}$ and $\mathrm{p} 16^{\mathrm{INK} 4 \mathrm{a}}$ tumor suppressor 
proteins. Loss-of-function of these proteins may promote the activation of cyclin-dependent kinase 4/6 (CDK4/6) via destabilization of p53 and inhibition of retinoblastoma (RB) protein (15), leading to acceleration of the G1-S transition and re-entry into the cell cycle (16). In addition, BRAF gene mutations have recently been shown to indicate a predisposition to melanoma (16). However, the detailed molecular mechanisms regarding the development of melanoma have remained poorly understood.

Recently, the effects of ASCs and their soluble factors on the viability of various types of tumor have been investigated; however, their effect has yet to be sufficiently addressed in melanoma cells. Therefore, the present study aimed to investigate whether ASC-conditioned medium (ASC-CM) treatment affected the proliferation and migration of B16 melanoma cells. In addition, the regulatory effect of ASC-CM on the tumorigensis of B16 melanoma cells was evaluated in a mouse model.

\section{Materials and methods}

ASC-CM preparation. ASCs were provided by Dr Jong-Hyuk Sung (Yonsei University, Seoul, Korea) (17). The cells $\left(4 \times 10^{5}\right)$ were seeded onto a 100-mm cell culture dish (BD Biosciences, Bedford, MA, USA) with the control medium [Dulbecco's modified Eagle's medium (DMEM) supplemented with $10 \%$ fetal bovine serum (FBS) (both from Invitrogen Life Technologies, Carlsbad, CA, USA)] and incubated overnight at $37^{\circ} \mathrm{C}$, prior to culture in DMEM/F-12 serum-free media (Gibco Life Technologies, Grand Island, NY, USA). Following three days of culture at $37^{\circ} \mathrm{C}$, ASC-CM was collected, centrifuged at a speed of $300 \mathrm{x}$ g for $5 \mathrm{~min}$ and filtered through a $0.22-\mathrm{mm}$ syringe filter (EMD Millipore, Bedford, MA, USA). The filtered ASC-CM was then applied to B16 melanoma cells (Korean Cell Line Bank, Seoul, Korea) at varying dilutions (0, $10,50$ and $100 \%)$.

Cell culture. B16 melanoma cells were cultured in a growth medium of DMEM containing $10 \%$ FBS and 1\% cellgro ${ }^{\circledR}$ penicillin/streptomycin solution (Mediatech, Inc., Manassas, VA, USA). The cells were grown in a humidified incubator at $37^{\circ} \mathrm{C}$ and $5 \% \mathrm{CO}_{2}$.

Cell proliferation assay. The B16 melanoma cells $\left(1 \times 10^{3}\right.$ cells/well) were cultured in 96 -well plates. Following one day of culture, the media was replaced with DMEM containing $10 \% \mathrm{FBS}$ and concentrated ASC-CM. Incubation with 10X ASC-CM occurred for varying time-periods (24, 48 and $72 \mathrm{~h}$ ), while incubation with various dilutions of ASC-CM (1, 5 and 10X) was performed for $72 \mathrm{~h}$. At the end of the indicated time-periods, cell proliferation was measured using a Cell Counting Kit-8 (CCK-8 kit; Dojindo Molecular Technologies, Inc., Gaithersburg, MD, USA), in accordance with the manufacturer's instructions. In brief, $10 \mu \mathrm{lCCK}-8$ solution was added to the cells and incubated for $3 \mathrm{~h}$. Absorbance was subsequently measured at a wavelength of $450 \mathrm{~nm}$ using a microplate reader (SpectraFluor, Tecan Austria GmbH, Grödig, Austria). The relative number of cells was calculated using comparable standard curves of the obtained optical density values.
Cell cycle analysis. The B16 melanoma cells were seeded in a medium containing $10 \% \mathrm{FBS}$ at $37^{\circ} \mathrm{C}$ and allowed to grow to $60 \%$ confluence. The cells were starved by incubation with serum-free medium for $24 \mathrm{~h}$, and were then continuously cultured for $24 \mathrm{~h}$ with or without 10X ASC-CM. Subsequently, the cells were harvested, washed twice with phosphate-buffered saline (PBS; Gibco-BRL, Carlsbad, CA, USA) and fixed in ice-cold $70 \%$ ethanol (Merck \& Co., Kenilworth, NJ, USA). The fixed cells were then washed once with PBS, incubated with RNase A (1 $\mu \mathrm{g} / \mu \mathrm{l}$; Qiagen, Inc., Valencia, CA, USA) for $1 \mathrm{~h}$ at $37^{\circ} \mathrm{C}$ and treated with propidium iodide (50 $\mu \mathrm{g} / \mathrm{ml}$; Sigma-Aldrich, St. Louis, MO, USA) for DNA staining. The distribution of the cell cycle phases was analyzed using a FACScan ${ }^{\mathrm{TM}}$ flow cytometer (BD Biosciences, San Jose, CA, USA).

Immunoblot analysis. Cell lysates were prepared in lysis

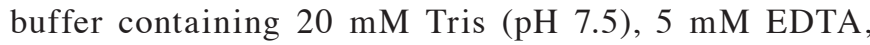
$10 \mathrm{mM} \mathrm{Na}_{4} \mathrm{P}_{2} \mathrm{O}_{7}, 100 \mathrm{mM} \mathrm{NaF}, 2 \mathrm{mM} \mathrm{Na}_{3} \mathrm{VO}_{4}, 1 \% \mathrm{NP}-40$ (all from Sigma-Aldrich), $1 \mathrm{mM}$ phenylmethylsulfonyl fluoride, $10 \mathrm{mg} / \mathrm{ml}$ aprotinin and $10 \mathrm{mg} / \mathrm{ml}$ leupeptin (ICN Biomedicals, Asse-Relegem, Belgium). The proteins were separated by $12 \%$ SDS-PAGE and then transferred onto nitrocellulose membranes (EMD Millipore) for immunoblot analysis. The membranes were incubated with rabbit polyclonal antibodies against CDK4 (IgG; catalog no. sc-260; Santa Cruz Biotechnology, Inc., Dallas, TX, USA), cyclin D1 (IgG; catalog no. 2407-0508; Bio-Rad AbD Serotec, Ltd., Kidlington, UK), p27 (IgG; catalog no. sc-528; Santa Cruz Biotechnology, Inc.), cyclin E (catalog no. 2925; Cell Signaling Technology, Inc., Danvers, MA, USA) and CDK2 (IgG; catalog no. sc-163; Santa Cruz Biotechnology, Inc.), with a goat polyclonal $\operatorname{IgG}$ anti- $\beta$-actin antibody (catalog no. sc-1615; Santa Cruz Biotechnology, Inc.) used as the internal control, at room temperature for $3 \mathrm{~h}$. All primary antibodies were used at a dilution of 1:1,000. The membranes were then incubated with goat anti-rabbit IgG (catalog no. sc-2004; Santa Cruz Biotechnology, Inc.) or donkey antigoat IgG (catalog no. sc-2020; Santa Cruz Biotechnology, Inc.) secondary antibodies conjugated with horseradish peroxidase, which were used at a dilution of 1:5,000, at room temperature for $1 \mathrm{~h}$. The bands were visualized with a ChemiDoc ${ }^{\mathrm{TM}}$ MP imaging system (Bio-Rad Laboratories, Inc., Hercules, CA, USA) and quantified using Image $\mathrm{Lab}^{\mathrm{TM}}$ software version 3.0 (Bio-Rad Laboratories, Inc.).

Cell migration analysis. Confluent B16 melanoma cells were maintained in serum-free medium for $24 \mathrm{~h}$ and a wound was formed using a razor blade. The cells were washed with PBS prior to the application of control medium or 10X ASC-CM. Images of the wounded area were captured at 12 and $24 \mathrm{~h}$ using phase-contrast microscopy (Eclipse TS100, Nikon Instruments, Inc., Melville, NY, USA). The wound closure was estimated by marking four randomly selected points along each wound and measuring the horizontal distance of the migrating cells from the initial wound.

Animals and xenograft model. Six-week-old C57BL/6 male mice $(n=20)$ were obtained from Orient Bio, Inc. (Seongnam, Korea) and acclimatized for one week; the animals were housed 
A

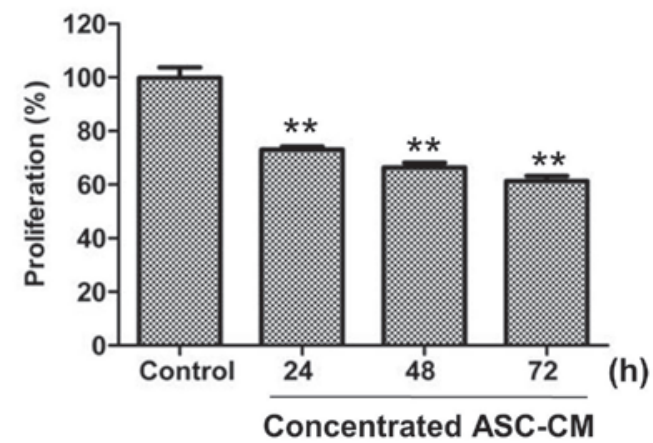

B

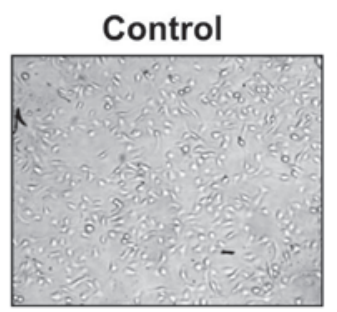

ASC-CM (10X)

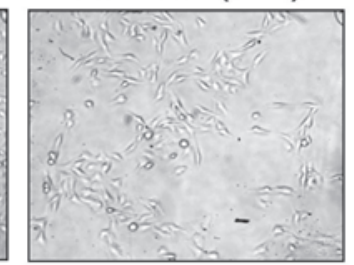

C

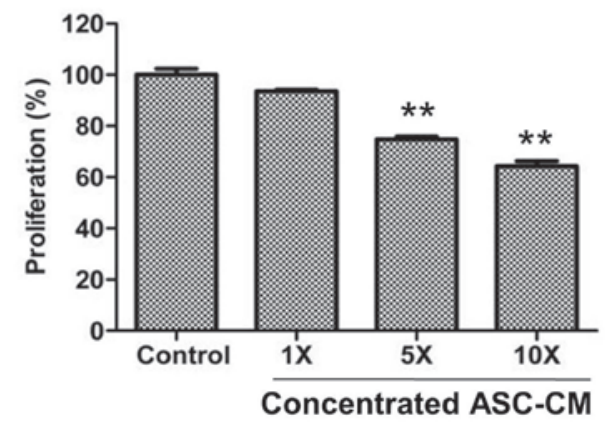

Figure 1. ASC-CM suppresses the proliferation of B16 melanoma cells. B16 melanoma cells were incubated in DMEM supplemented with 10\% FBS and ASC-CM at various concentrations. Control cells were incubated with DMEM (10\% FBS) alone for $72 \mathrm{~h}$. (A) Cells were treated with 10X ASC-CM for 24,48 and $72 \mathrm{~h}$ prior to measuring cell viability using a CCK-8 assay. (B) Following incubation with 10X ASC-CM for $72 \mathrm{~h}$, cell density was observed by phase-contrast microscopy (magnification, x100). (C) Cells were incubated with medium containing $1 \mathrm{X}, 5 \mathrm{X}$ and 10X ASC-CM for $72 \mathrm{~h}$, prior to measuring cell viability by performing a CCK- 8 assay. Values are presented as the mean \pm standard deviation. ${ }^{* *} \mathrm{P}>0.01$ vs. control. ASC-CM, adipose-derived stem cell-conditioned medium; DMEM, Dulbecco's modified Eagle's medium; FBS, fetal bovine serum; CCK-8, Cell Counting Kit-8.

in an air-conditioned room at $25^{\circ} \mathrm{C}$ with a $12 \mathrm{~h}$ dark/light cycle. All animals received human care with unlimited access to mouse chow and water. Animal experimental procedures were conducted in accordance with the guidelines of the INHA Institutional Animal Care and Use Committee of the Medical School of Inha University (Incheon, Korea). Mice were randomly divided into four groups ( $\mathrm{n}=5$; two control and two ASC-CM groups) and B16 melanoma cells $\left(1 \times 10^{6}\right)$ were inoculated into the dorsal flank of each mouse. When the tumors had reached a volume of $\sim 50-100 \mathrm{~mm}^{3}$, the mice were administered with a single intratumoral injection of 10X-concentrated ASC-CM (100 $\mu \mathrm{l})$ or vehicle (PBS; control group), daily or every other day $(n=5)$, a total of five times. Tumor axes were measured using calipers and tumor volume was calculated using the following equation: Volume $=0.5 \mathrm{x}$ long axis $\mathrm{x}(\text { short axis) })^{2}$. After 20 days, the mice were sacrificed by anaesthetizing with ketamine and rompun mixture, and their tumors were excised.

Immunohistochemistry. Tumor specimens were fixed with $10 \%$ buffered formaldehyde and embedded in paraffin (Sigma-Aldrich). Immunohistochemical staining was performed using $8 \mu \mathrm{m}$-thick sections of the tumor samples following deparaffinization, as previously described (18). Primary antibodies against Ki-67 (Abcam, Cambridge, UK) and CD34 (Santa Cruz Biotechnology, Inc., Santa Cruz, CA, USA) were applied. Unbound primary antibodies were washed off and the sections were incubated with a secondary biotinylated immunoglobulin $\mathrm{G}$ antibody. The stained cells were visualized using avidin-biotin complex $(\mathrm{ABC})$ solution followed by incubation with a 3,3-diaminobenzidine tetrahydrochloride solution using a Vectastain Elite ABC kit (Vector Laboratories, Inc., Burlingame, CA, USA).

Statistical analysis. Statistical analyses were performed using an unpaired Student's t-test. All data are presented as the mean \pm standard deviation and $\mathrm{P}<0.05$ was considered to indicate a statistically significant value.

\section{Results}

ASC-CM suppresses the proliferation of B16 melanoma cells. The present study initially investigated the effect of ASC-CM on the proliferation of B16 melanoma cells in vitro using a CCK-8 assay. Incubation of the B16 melanoma cells with 10X ASC-CM significantly suppressed cell proliferation in a time-dependent manner, compared with the control cells incubated in basal medium without ASC-CM $(\mathrm{P}<0.01$; Fig. 1A). Following a 72-h incubation period, cells treated with ASC-CM exhibited a $\sim 40 \%$ reduction in viability. In agreement with the CCK-8 findings, microscopic analysis identified retarded cell growth in 10X ASC-CM-treated cells compared with that of the control (Fig. 1B). Furthermore, the anti-proliferative effect of ASC-CM on melanoma cells was clarified by incubation with various concentrations of ASC-CM $(1,5$ and 10X), demonstrating a significant dose-dependent decrease in proliferation $(\mathrm{P}<0.05$; Fig. $1 \mathrm{C})$. These data indicated that culture medium from ASCs may effectively suppress the proliferation of B16 melanoma cells.

ASC-CM-induces cell cycle arrest in B16 melanoma cells at $G 1$ phase without induction of cell death. To elucidate the mechanism by which ASC-CM exerts its anti-proliferative effect, the present study analyzed the cell cycle of B16 melanoma cells following incubation with 10X ASC-CM for $24 \mathrm{~h}$. ASC-CM treatment significantly increased the number of cells in the $\mathrm{G} 1 / \mathrm{S}$ phase by $\sim 2.5$-fold $(\mathrm{P}<0.01)$, while reducing the proportion of cells in the $\mathrm{S}$ and $\mathrm{G} 2 / \mathrm{M}$ phases. These data indicated that the anti-proliferative effect of ASC-CM is induced by G1 phase arrest (Fig. 2A and B). Furthermore, no detectable difference in the proportion of cells in the sub-G1 phase was observed, indicating that ASC-CM-induced cell growth retardation is not associated with the induction of cell death. In addition, western blot analysis demonstrated that ASC-CM treatment downregulated cyclin D1 protein expression, which promotes G1/S transition, without detectable changes in the expression 

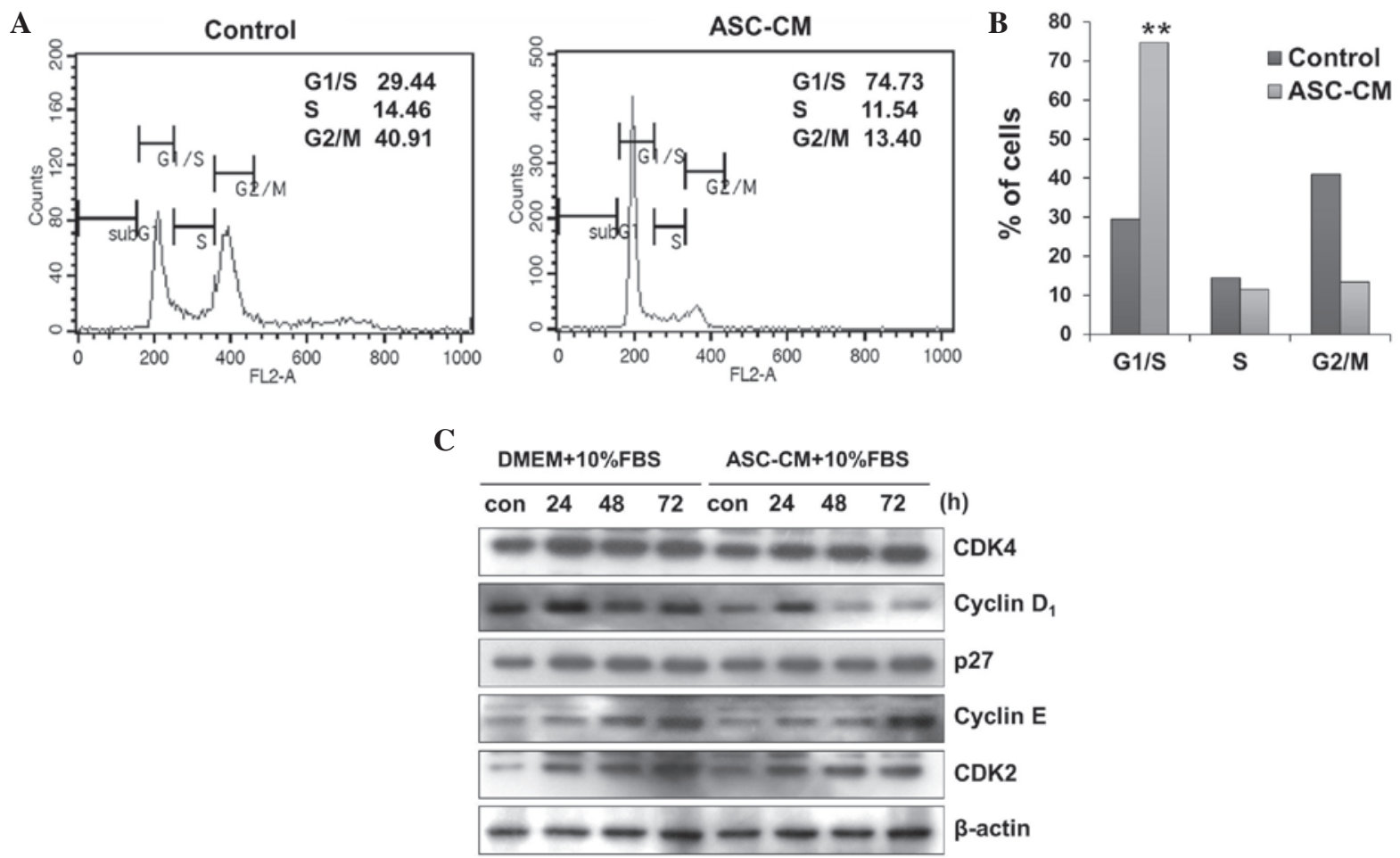

Figure 2. ASC-CM induces cell cycle arrest in B16 cells at G1 phase. Cell cycle analysis of B16 melanoma cells incubated with ASC-CM. (A) Cells were incubated with 10X ASC-CM for $24 \mathrm{~h}$ and stained with propidium iodide. Cell cycle distribution was analyzed by flow cytometry. (B) Relative number of cells in the G1/S , $\mathrm{S}$, and $\mathrm{G} 2 / \mathrm{M}$ phases were compared between the control group and the ASC-CM-treated cells. A representative result from three independent experiments is shown. ${ }^{* *} \mathrm{P}<0.01$ vs. control. (C) Cells were treated with or without ASC-CM (10X) and the protein expression levels of key cell cycle regulatory proteins, including cyclin D1, p27, cyclin E and CDK2, were determined by performing an immunoblot analysis. $\beta$-actin was used as the loading control. ASC-CM, adipose-derived stem cell-conditioned medium; DMEM, Dulbecco's modified Eagle's medium; FBS, fetal bovine serum; con, control; CDK, cyclin-dependent kinase.

A

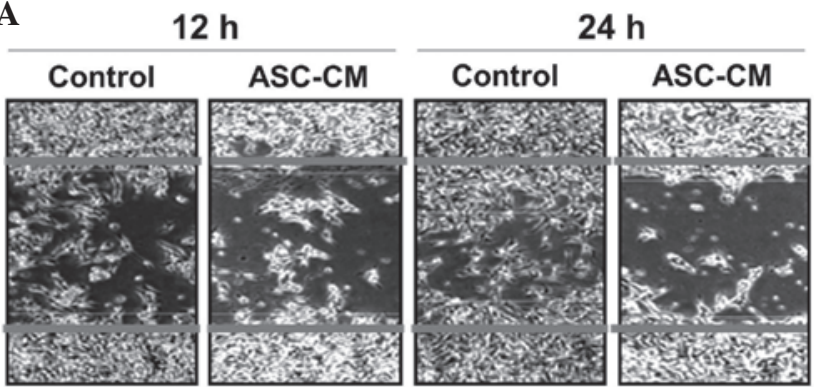

B

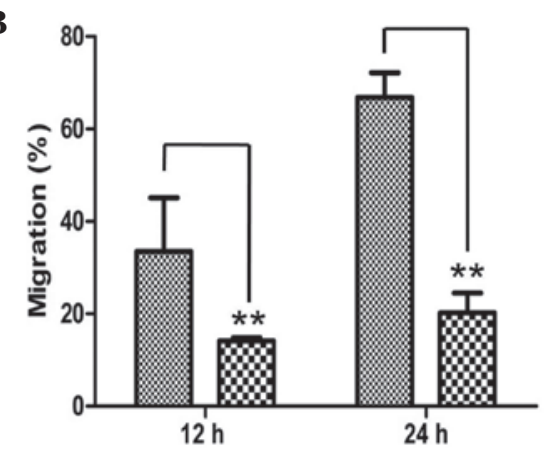

Figure 3. ASC-CM inhibits B16 melanoma cell migration. (A) Cells were grown to $90 \%$ confluence in $60-\mathrm{mm}$ diameter culture dishes prior to scratching with a razor blade. Cells were cultured in medium containing $10 \%$ fetal bovine serum alone (control) or ASC-CM. Cells were allowed to migrate for 12 and $24 \mathrm{~h}$ before migration was recorded by phase contrast microscopy. (B) Quantification of cell migration by counting the number of cells that moved beyond the reference line. Values are represented as the mean \pm standard deviation of three independent experiments. ${ }^{* *} \mathrm{P}<0.01$ vs. control. ASC-CM, adipose-derived stem cell-conditioned medium. of cyclin E or p27, a CDK inhibitor (Fig. 2C). Thus, ASC-CM appears to reduce the proliferation of B16 melanoma cells by inducing G1 arrest via modulation of cell cycle regulators.

ASC-CM decreases the migration of B16 melanoma cells. In addition to facilitating normal embryonic development, melanocyte migration is a critical step in the malignant transformation of metastatic melanomas. To determine whether ASC-CM was able to regulate the migration of B16 melanoma cells, a wound migration assay was conducted in B16 cells incubated with or without ASC-CM. Following wounding, the melanocytes were incubated for 12 or $24 \mathrm{~h}$ in medium containing ASC-CM. Subsequent phase contrast microscopy revealed that migration was markedly reduced in the ASC-CM-treated cells (Fig. 3A). Subsequently, migration was quantified by measuring the horizontal distance of the migrating cells from the initial wound. Under physiological conditions, cell migration was calculated as $\sim 30$ and $\sim 65 \%$ following incubation for 12 and $24 \mathrm{~h}$, respectively. However, migration was significantly retarded in the ASC-CM-treated cells, resulting in $<20 \%$ migration following 12 and $24 \mathrm{~h}$ of incubation $(\mathrm{P}<0.01$; Fig. 3B). The results of the present study indicate that, as well as reducing cell proliferation, ASC-CM is able to effectively suppress the migration of B16 melanoma cells.

ASC-CM suppresses B16 melanoma cell tumor growth in a mouse xenograft model. Considering the aforementioned in vitro findings, the effects of ASC-CM were investigated in an 
in vivo mouse transplantation model. Following subcutaneous injection of B16 melanoma cells ( $1 \times 10^{6}$ cells) into the right flank of C57BL/6 mice and the growth of a tumor mass to a volume of $\sim 50-100 \mathrm{~mm}^{3}, 100 \mu \mathrm{l}$ of $10 \mathrm{X}$ ASC-CM was administered intratumorally, daily or every other day, a total of five times. By measuring the tumor volume, it was identified that tumor growth was significantly retarded in the ASC-CM-treated groups compared with that of the control group following treatment daily or every other day, exhibiting a 71.4 and $73.7 \%$ reduction in size at day 18 , respectively $(\mathrm{P}<0.01 ; \mathrm{n}=5$ per group; Fig. 4A and B). In addition, immunohistochemistry revealed that the numbers of actively proliferating Ki-67 positive cells and CD34-positive endothelial cells were markedly decreased in the ASC-CM-treated group, indicating that ASC-CM exerts anti-tumorigenic activity in vivo (Fig. 4C). Collectively, these in vivo transplantation data support the hypothesis of a role for ASC-CM in the efficient inhibition of tumor growth in mice, as well as in an in vitro cell culture model.

\section{Discussion}

Adipose tissue consists of populations of heterogeneous cell types, including ASCs, endothelial cells, preadipocytes, pericytes, hematopoietic-lineage cells and fibroblasts, termed the SVF (19). ASCs are able to differentiate into mesodermal cell types (1), neuronal-like cells of ectodermal lineage (20-23) and cells of epidermal lineage (24), demonstrating their multi-lineage potential. This has resulted in the use of ASCs an alternative to pluripotent ESCs in the laboratory and for clinical use. A promising area for ASC therapeutics is in the regeneration of various tissues, including cardiovascular, bone, cartilage, nerve and liver tissues.

Previously, it was demonstrated that ASCs and ASC-CM have skin-regenerative potential $(4,17,25-28)$. Studies identified that direct ASC transplantation or the application of ASC-CM resulted in a significant acceleration in wound-healing, the promotion of hair regeneration, a reduction in undesirable pigmentation and anti-wrinkle effects $(4,17,25-28)$. These skin-regenerative results arise primarily from paracrine effects, including anti-apoptotic effects and angiogenesis of ASC secretory factors, in addition to their building-block function. Furthermore, ASCs and their soluble factors have been reported to increase the proliferation and migration of normal keratinocytes and fibroblasts $(4,17)$. Notably, ASC-CM administration appeared to inhibit melanin synthesis in B16 melanoma cells by downregulating tyrosinase and tyrosinase-related proteins (27). However, the effects of ASC-CM on cell proliferation and migratory ability have yet to be sufficiently addressed. The present study aimed to investigate whether ASC-CM treatment affected the proliferation and migration of B16 melanoma cells and evaluate the ability of ASC-CM to regulate a B16 melanoma cell tumor mass in a mouse xenograft model.

To date, only a small number of studies have described the molecular mechanisms underlying the tumorigenesis of melanoma. Linkage analysis on melanoma families revealed that the development of melanoma may be initiated by loss-of-function mutations in the two tumor suppressor proteins, $\mathrm{p} 14^{\mathrm{ARF}}$ and $\mathrm{p} 16^{\mathrm{INK4a}}$, alternative splicing products coded by the CDKN2A gene (29). p14 ${ }^{\mathrm{ARF}}$ stabilizes p53 by rapidly degrading mouse/human double minute-2 $(30,31)$. By contrast, p16 ${ }^{\text {INK4a }}$

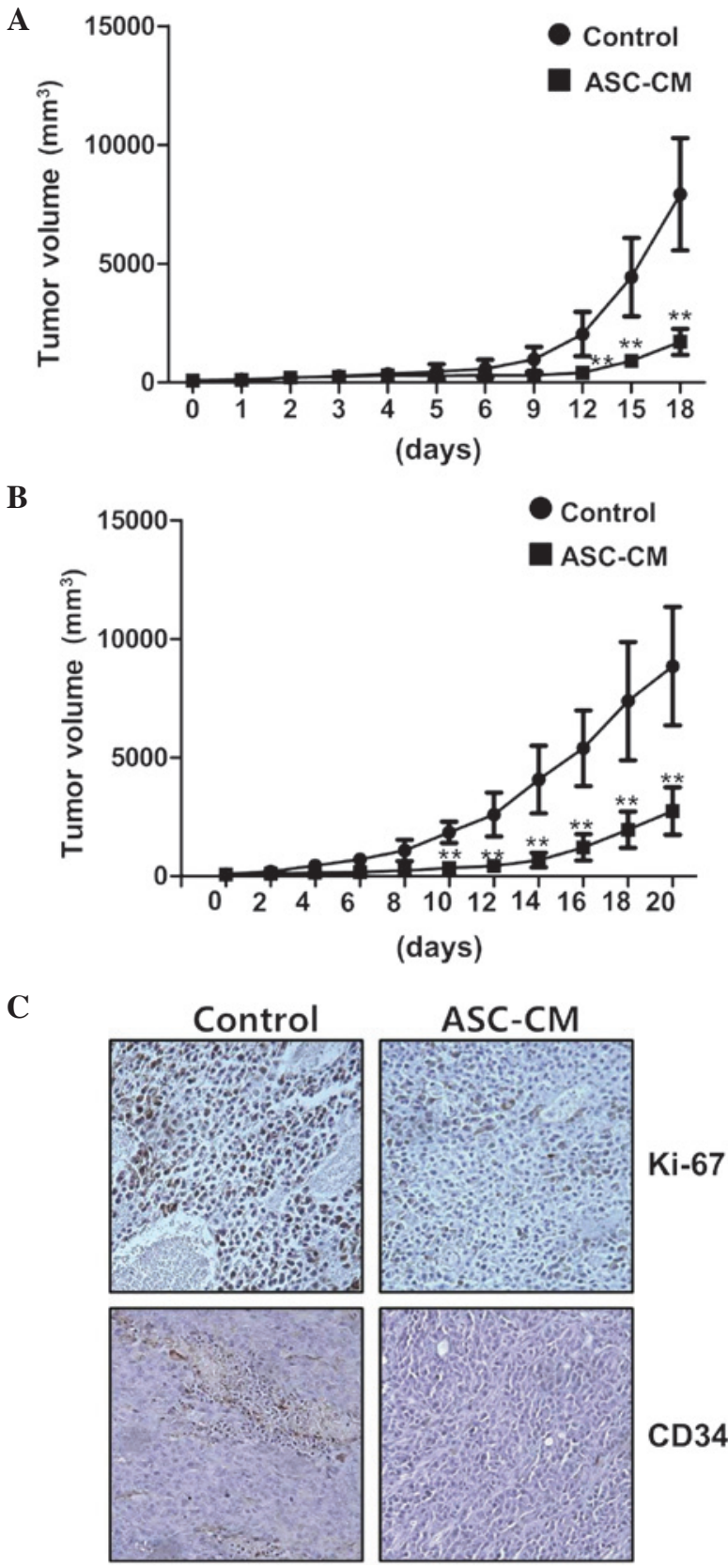

Figure 4. ASC-CM suppresses tumor growth in a mouse xenograft model. Following subcutaneous injection of B16 melanoma cells $\left(1 \times 10^{6}\right.$ cells $)$ into C57BL/6 mice, an intratumoral injection of 10X ASC-CM $(100 \mu 1)$ was administered (A) daily or (B) every other day, for a total of five times. Tumor size was measured using calipers and tumor volume calculated as $0.5 \mathrm{x}$ long axis $\mathrm{x}$ (short axis) ${ }^{2}$. (C) Immunohistochemical analysis of Ki-67 and CD34 protein expression in the isolated tumors xenografts of control and ASC-CM-treated mice (magnification, $\mathrm{x} 400$ ). $\mathrm{n}=5$ per group. ${ }^{* *} \mathrm{P}<0.01$ vs. control. ASC-CM, adipose-derived stem cell-conditioned medium; CD34, cluster of differentiation 34

enhances binding of RB protein with transcription factors of the E2F family by suppressing CDK4/6 activity (15). Mutations in $\mathrm{p} 14^{\mathrm{ARF}}$ and $\mathrm{p} 16^{\mathrm{INK} 4 \mathrm{a}}$ proteins may promote G1-S phase transition, as well as re-entry into the cell cycle, thus, potentiating abnormal cell proliferation (16). In addition, BRAF gene mutations have recently been identified to indicate a predisposition to melanoma. Furthermore, the mitogen-activated protein kinase and phosphatidylinositide 3-kinase signaling pathways are also activated in the transcription of critical cell proliferation genes in the 
majority of cases of melanoma (32). In the present study, it was identified that treatment with concentrated ASC-CM inhibited the proliferation of B16 melanoma cells by $\sim 40 \%$ and that this anti-proliferative effect was induced by cell cycle arrest at the G1 phase, as revealed by flow cytometry. This cell cycle arresting effect of ASC-CM was clarified by the detection of decreased cyclin D1 expression levels, indicating that there may be merit in the development of ASC-CM into a therapeutic agent in the future. The present study also performed a wound migration assay, which indicated that the migration of melanocytes was inhibited following treatment with ASC-CM, indicating that ASC-CM exhibited an anti-tumorigenic effect on the transformed melanocytes. In addition to these in vitro evidences, ASC-CM was demonstrated to decrease tumor growth in a mouse model, indicating that ASCs or ASC-CM may be promising candidates for future therapeutic strategies targeting melanoma.

ASCs produce various growth factors and adipokines, thus, the cells function as an endocrine or paracrine organ that is considered to perform a pivotal role in maintaining a suitable microenvironment for the regulation of tumor initiation, progression and metastasis (27,33-35). ASCs express a number of biological proteins involved in the promotion of cell viability, including transforming growth factor $\beta 1(27,33)$ and progranulin (34). However, numerous studies have demonstrated the anti-proliferative or anti-tumorigenic effects of ASCs. For example, preconditioned endothelial progenitor cells have been observed to reduce melanoma metastasis by cell-cell interactions induced by secreted protein, acidic and rich in cysteine, as well as endocytosis (9). Human ESCs have been reported to secrete soluble factors that arrest the growth of human epithelial cancer cell lines (10). For example, angiopoietin-1 produced from ASCs appears to exhibit an inhibitory effect on neointimal formation in the rat femoral artery (36). However, the profile of cytokines released from ASCs may be modulated by external factors; for example, hepatocyte growth factor was released from ASCs following an exposure to basic fibroblast grow th factor or epidermal growth factor (EGF), while ASCs secreted various hematopoietic factors and proinflammatory cytokines following lipopolysaccharide treatment (37). These results indicate that environmental factors are crucial for determination of the proteins that are released from ASCs. Furthermore, a recent study demonstrated that varying expression levels of protein factors are released in accordance with ASC subtype. Specifically, monocyte chemotactic protein-1, eotaxin, IL-1 receptor agonist, IL-6, granulocyte-macrophage colony-stimulating factor and vascular EGF were more abundant in conditioned medium obtained from the visceral omental ASC fraction compared with that of the abdominal subcutaneous ASC fraction, indicating heterogeneity in the secretory ability of visceral and subcutaneous fat tissue (35). These findings indicate that the set of factors secreted from ASCs may be determined by the cell origin and the environmental factors during the culturing process.

Controversy remains surrounding the interpretation of studies exploring the induction versus inhibition of cancer following stem cell transplantation, therefore, hindering their clinical use. Despite the controversial interpretations regarding their effects on various types of cancer, freshly isolated ASCs and cosmetics using ASC-CM are commercially available and are currently undergoing clinical trials in certain countries.
In conclusion, the present study shows that ASC-CM attenuates the proliferation and migration of B16 melanoma cells in culture and in a mouse xenograft model, suggesting ASC-CM may be a useful material for anti-cancer therapeutics. Further knowledge must be acquired regarding the safety of ASCs and ASC-CM prior to their use in future clinical applications.

\section{Acknowledgements}

The present study was supported by grants from the Korean Health Technology Research and Development Project of the Korean Ministry of Health and Welfare (grant no. A120266) and from Inha University.

\section{References}

1. Zuk PA, Zhu M, Mizuno H, et al: Multilineage cells from human adipose tissue: Implications for cell-based therapies. Tissue Eng 7: 211-228, 2001.

2. Hokugo A, Sorice S, Parhami F, et al: A novel oxysterol promotes bone regeneration in rabbit cranial bone defects. J Tissue Eng Regen Med: Aug 29, 2013 (Epub ahead of print).

3. Fu X, Fang L, Li H, et al: Adipose tissue extract enhances skin wound healing. Wound Repair Regen 15: 540-548, 2007.

4. Kim WS, Park BS, Sung JH, et al: Wound healing effect of adipose-derived stem cells: A critical role of secretory factors on human dermal fibroblasts. J Dermatol Sci 48: 15-24, 2007.

5. Yu JM, Jun ES, Bae YC and Jung JS: Mesenchymal stem cells derived from human adipose tissues favor tumor cell growth in vivo. Stem Cells Dev 17: 463-473, 2008.

6. Prantl L, Muehlberg F, Navone NM, et al: Adipose tissue-derived stem cells promote prostate tumor growth. Prostate 70: 1709-1715, 2010

7. Eterno V, Zambelli A, Pavesi L, et al: Adipose-derived Mesenchymal Stem Cells (ASCs) may favour breast cancer recurrence via HGF/c-Met signaling. Oncotarget 5: 613-633, 2014.

8. Koellensperger E, Gramley F, Preisner F, Leimer U, Germann G and Dexheimer V: Alterations of gene expression and protein synthesis in co-cultured adipose tissue-derived stem cells and squamous cell-carcinoma cells: Consequences for clinical applications. Stem Cell Res Ther 5: 65, 2014.

9. Rowan BG, Gimble JM, Sheng M, et al: Human adipose tissue-derived stromal/stem cells promote migration and early metastasis of triple negative breast cancer xenografts. PLoS One 9: e89595, 2014.

10. Sun B, Roh KH, Park JR, et al: Therapeutic potential of mesenchymal stromal cells in a mouse breast cancer metastasis model. Cytotherapy 11: 289-298, 2009.

11. Cousin B, Ravet E, Poglio S, et al: Adult stromal cells derived from human adipose tissue provoke pancreatic cancer cell death both in vitro and in vivo. PLoS One 4: e6278, 2009.

12. Takahara K, Ii M, Inamoto T, et al: Adipose-derived stromal cells inhibit prostate cancer cell proliferation inducing apoptosis. Biochem Biophys Res Commun 446: 1102-1107, 2014.

13. National Cancer Institute: Surveillance, Epidemiology, and End Results Program. SEER Stat Fact Sheets: Melanoma of the Skin. http://seer.cancer.gov/statfacts/html/melan.html. Accessed April 25, 2015.

14. Squires MH III and Delman KA: Current treatment of locoregional recurrence of melanoma. Curr Oncol Rep 15: 465-472, 2013.

15. Koh J, Enders GH, Dynlacht BD and Harlow E: Tumour-derived p16 alleles encoding proteins defective in cell-cycle inhibition. Nature 375: 506-510, 1995.

16. Sheppard KE and McArthur GA: The cell-cycle regulator CDK4: an emerging therapeutic target in melanoma. Clin Cancer Res 19: 5320-5328, 2013

17. Won $\mathrm{CH}$, Yoo HG, Kwon OS, et al: Hair growth promoting effects of adipose tissue-derived stem cells. J Dermatol Sci 57: 134-137, 2010.

18. Ma YL, Peng JY, Zhang P, Liu WJ, Huang L and Qin HL: Immunohistochemical analysis revealed CD34 and Ki67 protein expression as significant prognostic factors in colorectal cancer. Med Oncol 27: 304-309, 2010. 
19. Kokai LE, Marra K and Rubin JP: Adipose stem cells: Biology and clinical applications for tissue repair and regeneration. Transl Res 163: 399-408, 2014

20. Safford KM, Safford SD, Gimble JM, Shetty AK and Rice HE: Characterization of neuronal/glial differentiation of murine adipose-derived adult stromal cells. Exp Neurol 187: 319-328, 2004

21. Ashjian PH, Elbarbary AS, Edmonds B, et al: In vitro differentiation of human processed lipoaspirate cells into early neura progenitors. Plast Reconstr Surg 111: 1922-1931, 2003.

22. Kingham PJ, Kalbermatten DF, Mahay D, Armstrong SJ, Wiberg $\mathrm{M}$ and Terenghi G: Adipose-derived stem cells differentiate into a Schwann cell phenotype and promote neurite outgrowth in vitro. Exp Neurol 207: 267-274, 2007.

23. Xu Y, Liu L, Li Y, et al: Myelin-forming ability of Schwann cell-like cells induced from rat adipose-derived stem cells in vitro. Brain Res 1239: 49-55, 2008.

24. Trottier V, Marceau-Fortier G, Germain L, Vincent C and Fradette J: IFATS collection: Using human adipose-derived stem/stromal cells for the production of new skin substitutes Stem Cells 26: 2713-2723, 2008.

25. Kim WS, Park BS, Kim HK, et al: Evidence supporting antioxidant action of adipose-derived stem cells: Protection of human dermal fibroblasts from oxidative stress. J Dermatol Sci 49: 133-142, 2008

26. Kim WS, Park BS, Park SH, Kim HK and Sung JH: Antiwrinkle effect of adipose-derived stem cell: Activation of dermal fibroblast by secretory factors. J Dermatol Sci 53: 96-102, 2009.

27. Kim WS, Park SH, Ahn SJ, et al: Whitening effect of adipose-derived stem cells: A critical role of TGF-beta 1. Biol Pharm Bull 31: 606-610, 2008.

28. Kim WS, Park BS and Sung JH: The wound-healing and antioxidant effects of adipose-derived stem cells. Expert Opin Biol Ther 9: 879-887, 2009.
29. Tsao H, Chin L, Garraway LA and Fisher DE: Melanoma: From mutations to medicine. Genes Dev 26: 1131-1155, 2012.

30. Kamijo T, Weber JD, Zambetti G, Zindy F, Roussel MF and Sherr CJ: Functional and physical interactions of the ARF tumor suppressor with p53 and Mdm2. Proc Natl Acad Sci USA 95: 8292-8297, 1998

31. Zhang Y, Xiong $\mathrm{Y}$ and Yarbrough WG: ARF promotes MDM2 degradation and stabilizes p53: ARF-INK4a locus deletion impairs both the $\mathrm{Rb}$ and $\mathrm{p} 53$ tumor suppression pathways. Cell 92: 725-734, 1998.

32. Eggermont AM, Spatz A and Robert C: Cutaneous melanoma. Lancet 383: 816-827, 2014.

33. Kim BS, Kang KS and Kang SK: Soluble factors from ASCs effectively direct control of chondrogenic fate. Cell Prolif 43: 249-261, 2010.

34. Tsuruma K, Yamauchi M, Sugitani S, et al: Progranulin, a major secreted protein of mouse adipose-derived stem cells, inhibits light-induced retinal degeneration. Stem Cells Transl Med 3: 42-53, 2014

35. Perrini S, Ficarella R, Picardi E, et al: Differences in gene expression and cytokine release profiles highlight the heterogeneity of distinct subsets of adipose tissue-derived stem cells in the subcutaneous and visceral adipose tissue in humans. PLoS One 8: e57892, 2013

36. Takahashi M,Suzuki E, Kumano S, et al: Angiopoietin-1 mediates adipose tissue-derived stem cell-induced inhibition of neointimal formation in rat femoral artery. Circ J 77: 1574-1584, 2013.

37. Kilroy GE, Foster SJ, Wu X, et al: Cytokine profile of human adipose-derived stem cells: Expression of angiogenic, hematopoietic, and pro-inflammatory factors. J Cell Physiol 212: 702-709, 2007. 